\title{
The Effect of Surface Roughness of a Body in the High Reynolds-Number Flow
}

\author{
TSUTOMU ADACHI \\ Faculty of Engineering, Osaka Sangyo University, 3-1-1, Nakagaito, Daito, Osaka, Japan (574)
}

\begin{abstract}
In this paper, first, the principle, structures, operations, and performances of the cryogenic wind tunnel are described. By changing the pressure, temperature and velocity of gas a high Reynolds-number flow $\left(5 \times 10^{4}<R_{e}<10^{7}\right)$ can be obtained. From the research results, a high Reynolds-number flow with comparatively low power, $\mathrm{LN}_{2}$ consumptions was attained. It was with Mach-number independent of each other. o show some examples of high Reynolds-number flow, the effects of surface roughness and grooves on the surface of a cylinder on the flow are measured using models with various values of roughness and size. A model test of an airship was also conducted. With the high Reynolds-number flow, the thickness of the boundary layer becomes thinner. Then the surface conditions of a body have great effect on the flow phenomena and on the drag of the body. Some attempts to reduce the drag of the body were shown.
\end{abstract}

Key Words: High Reynolds number; Surface roughness; Boundry layer; Drag; Strouhal number

\section{INTRODUCTION}

$\mathbf{I}^{\mathrm{n}}$ the flow around a large structure or high speed vehicles, the Reynolds-number becomes large. In order to do wind tunnel measurements around bodies in a stream with a geometrically similar model, the Reynolds-number must have the same value for the model and for the prototype. Using the cryogenic wind tunnel we can get a high Reynolds-number flow by decreasing the temperature and making the pressure high. As we can make pressure high in the wind tunnel, we can get high a Reynolds-number flow at a comparatively low Machnumber. In this paper, first the special features of our cryogenic wind tunnel are described.

Effects of the Reynolds-number on the flow of a body placed in a stream raise important problems because the thickness of the boundary layers becomes thinner in high Reynolds-number ranges. For example, an increase in the Reynolds-number drag coefficient of a cylinder decreases at the critical point and thereafter increases in the supercritical Reynolds-number range. The flow phenomena have not been made clear because there have not been so many apparatuses for high Reynolds-number flow. The flow phenomena around a cylinder vary with the Reynolds-number and they are influenced by the surface conditions. Then the drag coefficient and Strouhal-number vary with the Reynolds-number and surface roughness. First the effects of surface roughness of a circular cylinder with a wide range of Reynolds-number are measured with 8 cylinders with various homogeneous surface roughnesses. It was made clear that if the roughness was not homogenous its roughness was greater in the axial direction than in the circumferential direction, i.e., if grooves were engraved around a cylinder, its drag coefficient showed some decrease in the critical point and in the supercritical Reynolds-number range. Effects of the surface grooves on the drag and Strouhal number were measured and showed some decreases in drag, where the sizes of the grooves were determined by the reference results of research on the grooves along a flat plate placed parallel to the stream, i.e., a riblet.

There is a plan for making an airship stay at one point in the stratosphere. In order to make it stay at one point, the drag on the body must be small. The flow around the body are high Reynolds and low Mach-number flows. It was shown that its drag can be made smaller if suction is applied to the boundary layer.

\section{SPECIAL CHARACTERISTIC OF THE CRYOGENIC WIND TUNNEL}

The specifications and general view of our cryogenic wind tunnel are shown in Table 1 and Fig. 1. The outer 
TABLE 1

Specifications and main dimensions of the cryogenic wind tunnel

\begin{tabular}{ll}
\hline Type of Wind tunnel & Göttingen Type Inner insulated \\
Pressure & $0.8 \mathrm{MPa}$ \\
Temperature & $112 \mathrm{~K}$ \\
Maximum Velocity & $65 \mathrm{~m} / \mathrm{s}$ \\
Maximum Reynolds Number & $10^{7}$ \\
Maximum Mach Number & 0.3 \\
Velocity Distribution & Lower than $\pm 1 \%$ \\
Turbulence & $0.26 \%($ Atmospheric Temp., $0.1 \mathrm{MPa}$, \\
& $34 \mathrm{~m} / \mathrm{s})$ \\
& $0.37 \%(110 \mathrm{~K}, 0.2 \mathrm{MPa}, 38 \mathrm{~m} / \mathrm{s})$ \\
\hline
\end{tabular}

surface was kept at atmospheric temperature in spite of the cryogenic temperature on the inside. Insulating materials, i.e., rigid urethane, glass wool, plywood, veneer board, etc., were placed between the inner and outer surfaces. The test section had a cross-section of $500 \times$ $500 \mathrm{~mm}$. By changing the velocity, pressure and temperature of the gas, a flow of arbitrary Reynolds-numbers was independently attained as shown in Fig. 2. As the maximum value of pressure in the tunnel was up to 8 $\mathrm{MPa}$, a high Reynolds-number flow with a low Machnumber was attained. For operation, first the pressure was increased and then the temperature was decreased. It took about 30 minutes for both of those processes respectively. Then the design conditions were obtained. These conditions were measured for 30 minutes [Adachi, et al. (1990a, 1991)].

\section{SOME EXAMPLES OF HIGH REYNOLDS- NUMBER FLOW EXPERIMENTS}

\section{Effects of the Surface Roughness on the Drag and the Strouhal-Number of a Cylinder}

An experimental investigation of the effects of the relative roughness was carried for the Reynolds-number

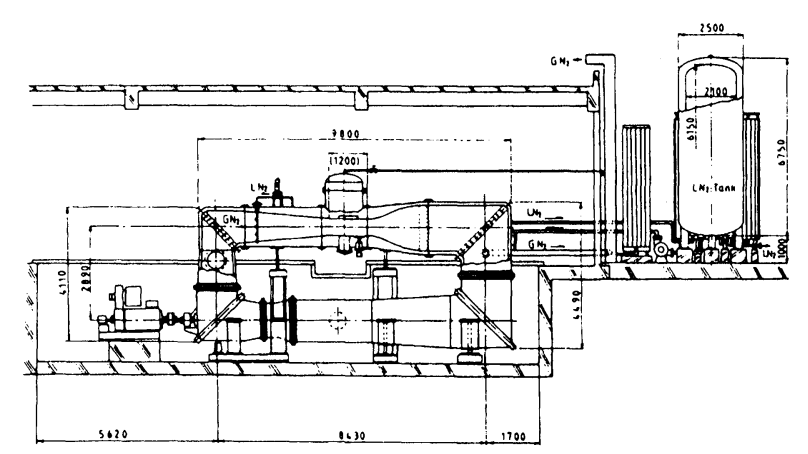

FIGURE 1 The general view of the cryogenic wind tunnel.

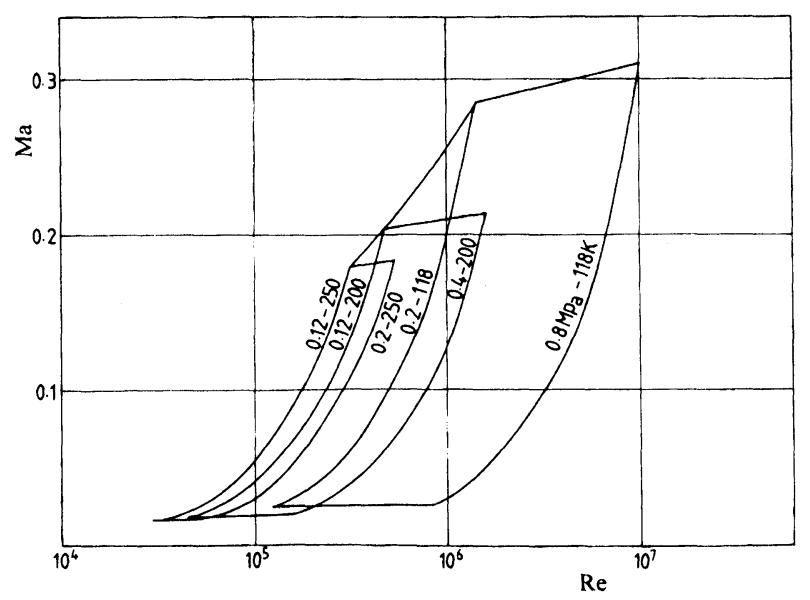

FIGURE 2 The Reynolds and Mach-numbers in the operation range.

ranges $5 \times 10^{4} \leqq R_{e} \leqq 10^{7}$ (Adachi, et al. 1989a, b, 1990b). 8 cylinders $50 \mathrm{~mm}$ in diameter were used for this purpose. Table 2 shows the values of surface roughness of these cylinders. Fig. 3 shows variations of drag coefficient $C_{d}$ with the Reynolds-number for various values of relative roughnesses. The flow behavior around a cylinder placed in the test section was classified into 4 regions irrespective of relative roughness. They are subcritical, transitional, supercritical and transcritical Reynolds-number ranges. These ranges were classified by the variations of the drag coefficient and Strouhalnumber. Indeed, the extent of the change became smaller with an the increase in the relative surface roughness $\left(K_{s} / d\right)$, but the appearance of variation had some similarities.

The variations of the drag coefficient with the back pressure coefficient could be summarized into two lines of different inclinations, as shown in Fig. 4. In the subcritical Reynolds-number range, the relation moved on the solid line in the direction of smaller absolute values of $C_{d}$ with an increase in the Reynolds-number. Then it transferred to the dotted line. It moved on this line in direction of a smaller value of $C_{p h}$ with an increase in the Reynolds-number. It took the minimum absolute value of $C_{d}$ at the critical point. Then it moved on the dotted line in the opposite direction. The shifting point between the transitional and supercritical Reynolds-number ranges is called the critical point. At this point the drag coefficient had its the minimum value. On the other hand, the Strouhal-number had its maximum value.

The value of relative roughness affected the Reynoldsnumber range in which these four ranges exist. In the subcritical range, the drag coefficient was 1.2 and the Strouhal-number was 0.2 , irrespective of surface rough- 
TABLE 2

Surface roughness of the cylinders

\begin{tabular}{|c|c|c|c|c|c|c|c|c|}
\hline & A & B & $\mathrm{C}$ & D & $\mathrm{E}$ & $\mathrm{F}$ & $\mathrm{G}$ & $\mathrm{H}$ \\
\hline roughness & $(0.08$ & $(0.3$ & $(10$ & $(20$ & $(50$ & $(70$ & $(50$ & (130 \\
\hline$K_{s t}(m)$ & $\begin{array}{l}\backsim 0.5) \\
\times 10^{-6}\end{array}$ & $\begin{array}{l}\sim 0.5) \\
\times 10^{-6}\end{array}$ & $\begin{array}{l}\sim 17) \\
\times 10^{-6}\end{array}$ & $\begin{array}{l}\sim 30) \\
\times 10^{-6}\end{array}$ & $\begin{array}{l}\text { ^68) } \\
\times 10^{-6}\end{array}$ & $\begin{array}{l}\text { ^90) } \\
\times 10^{-6}\end{array}$ & $\begin{array}{l}\backsim 110) \\
\times 10^{-6}\end{array}$ & $\begin{array}{l}\sim 150 \text { ) } \\
\times 10^{-6}\end{array}$ \\
\hline roughness & $(0.03$ & $(0.9$ & $(9$ & $(28$ & $(60$ & $(78$ & $(62$ & (119 \\
\hline$K_{s a}(m)$ & $\begin{array}{l}\sim 0.5) \\
\times 10^{-6}\end{array}$ & $\begin{array}{l}\sim 1.4) \\
\times 10^{-6}\end{array}$ & $\begin{array}{l}\sim 15) \\
\times 10^{-6}\end{array}$ & $\begin{array}{l}\sim 36) \\
\times 10^{-6}\end{array}$ & $\begin{array}{l}\sim 75) \\
\times 10^{-6}\end{array}$ & $\begin{array}{l}\sim 94) \\
\times 10^{-6}\end{array}$ & $\begin{array}{l}\sim 110) \\
\times 10^{-6}\end{array}$ & $\begin{array}{l}\sim 126) \\
\times 10^{-6}\end{array}$ \\
\hline $\begin{array}{l}\text { ratio } \\
\left(K_{s t} / K_{s a}\right)\end{array}$ & 0.85 & 0.38 & 1.08 & 0.75 & 0.84 & 0.89 & 0.98 & 1.03 \\
\hline $\begin{array}{l}\text { relative } \\
\text { roughness } \\
\left(K_{s} / d\right)\end{array}$ & $\begin{array}{l}4.54 \\
\times 10^{-6}\end{array}$ & $\begin{array}{l}8.57 \\
\times 10^{-6}\end{array}$ & $\begin{array}{l}254 \\
\times 10^{-6}\end{array}$ & $\begin{array}{l}500 \\
\times 10^{-6}\end{array}$ & $\begin{array}{l}1160 \\
\times 10^{-6}\end{array}$ & $\begin{array}{l}1600 \\
\times 10^{-6}\end{array}$ & $\begin{array}{l}1810 \\
\times 10^{-6}\end{array}$ & $\begin{array}{l}2540 \\
\times 10^{-6}\end{array}$ \\
\hline
\end{tabular}

ness. The shifting point between these Reynolds-number ranges moved towards the lower Reynolds-number with an increase in $\left(K_{s} / d\right)$. Fig. 5 shows variations of the minimum value of drag coefficient $C_{d \text { min }}$, i.e., the drag coefficient at the critical point, with relative surface roughness $\left(K_{s} / d\right)$. It is clear from this figure that $C_{d \text { min }}$ decreased with a decrease in $\left(K_{s} / d\right)$, but it became constant for the values smaller than $\left(K_{s} / d\right)=5 \times 10^{-4}$. Fig. 6 also shows the variation of the Reynolds-number at the critical point, i.e., $\operatorname{Re}\left(C_{d \text { min }}\right)$ with $\left(K_{s} / d\right)$. From these figures it became clear that there was a limiting value of surface roughness $\left(K_{s} / d\right)$, which influenced the value of the Reynolds-number at the critical point. If the relative surface roughness was smaller than this limiting value, the variations of $C_{d}$ with the Reynolds-number showed the same trend. This limiting value is called the critical surface roughness. Its value is $\left(K_{s} / d\right)_{\text {crit }}=5 \times$ $10^{-4}$. For a cylinder with a larger surface roughness than $\left(K_{s} / d\right)_{c r i t}$, the Reynolds-number at the critical point became smaller with an increase in $\left(K_{s} / d\right)$. Fig. 7 shows variations of drag coefficient $C_{d}$ with relative roughness

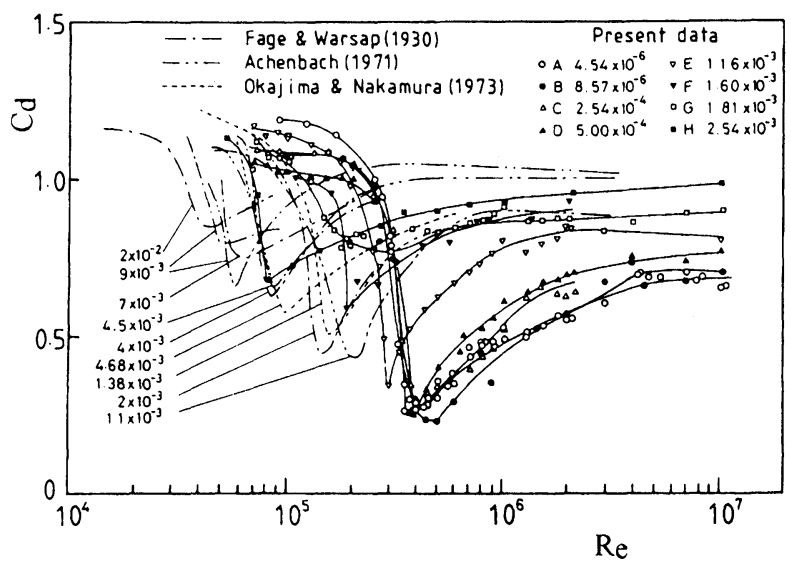

FIGURE 3 Variations of the drag coefficient with the Reynoldsnumber.
$\left(K_{s} / d\right)$ in the transcritical Reynolds-number range. The drag coefficient decreased with a decrease in the surface roughness in the transcritical Reynolds-number range. No limiting values could be seen. Then the flow phenomena in the super and transcritical Reynolds-number ranges were influenced by the surface roughness.

\section{The Universal Strouhal-Number in the Wide Range of Reynolds-Number Flows}

The universality of the Strouhal-number in the wake of a cylinder was considered. The universal Strouhal-numbers considered were Roshko's number (Roshko 1961), Bearman's number (Bearman 1967), Griffin's-number (Griffin 1981) and that took the measured values of the width of the wake vortex streets for the reference length. These universal Strouhal-numbers were calculated using the measured values of vortex frequency, surface pressure distributions and drag coefficients. Among these, the Bearman's number, which showed the most uniform

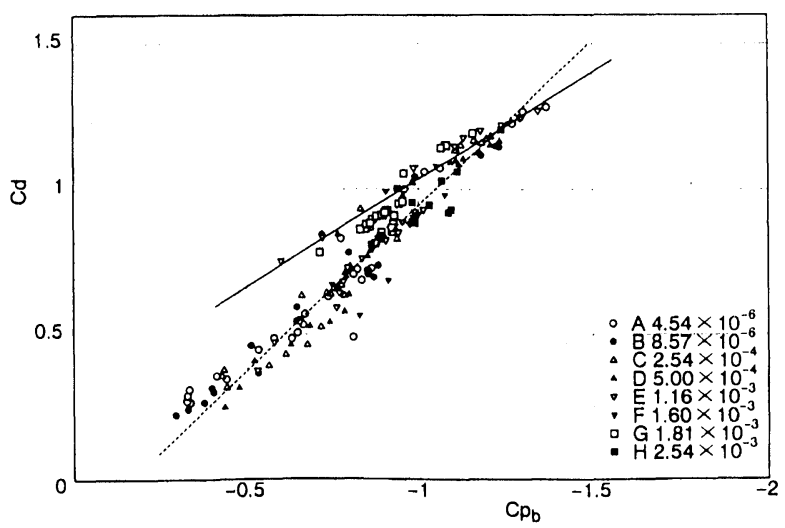

FIGURE 4 Variations of the drag coefficient with the pressure coefficient. 


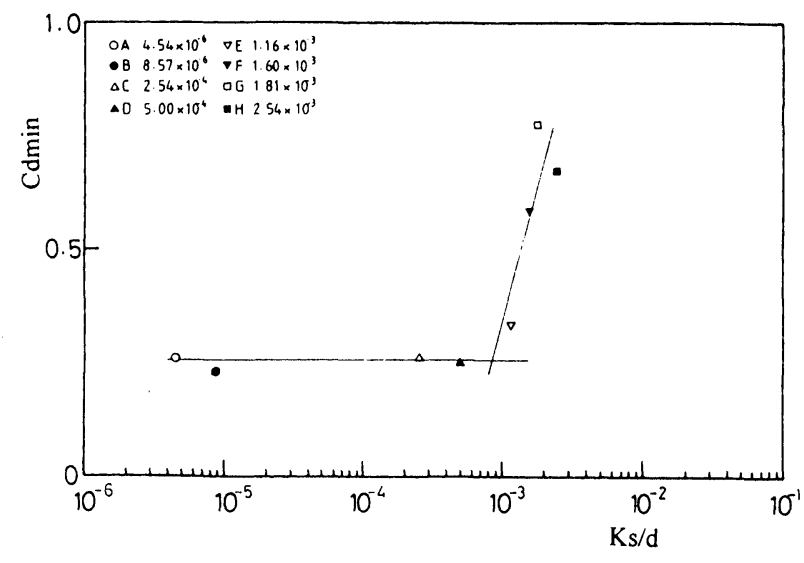

FIGURE 5 Variations of minimum drag coefficient $C_{d \min }$ ) with relative surface roughness $\left(K_{s} / d\right)$.

value throughout the Reynolds-number range was described.

Bearman's number introduced by Bearman is defined as:

$S_{B}=\frac{f h}{U_{s}}$

where $U_{s}$ is the velocity of the irrotational flow at the point of separation and is assumed to be expressed by the relationship

$$
U_{s}=k U_{\infty}=\left(1-C_{p b}\right)^{1 / 2}
$$

which may be obtained by applying Bernoulli's Equation to the flow just outside the boundary layer at separation.

Bearman proposed to evaluate $h$ by means of Kronauer's criterion, which states that the ratio of the lateral to the longitudinal spacing of the vortices, $(h / l)$, must be such that the potential vortex street drag coefficient $C_{D s}$ is at a minimum. $C_{D s}$ is defined as $C_{D s}=D_{s} /\left(\rho U_{\infty}^{2} l / 2\right)$, where $D_{s}$ is the vortex street drag. At this point, the

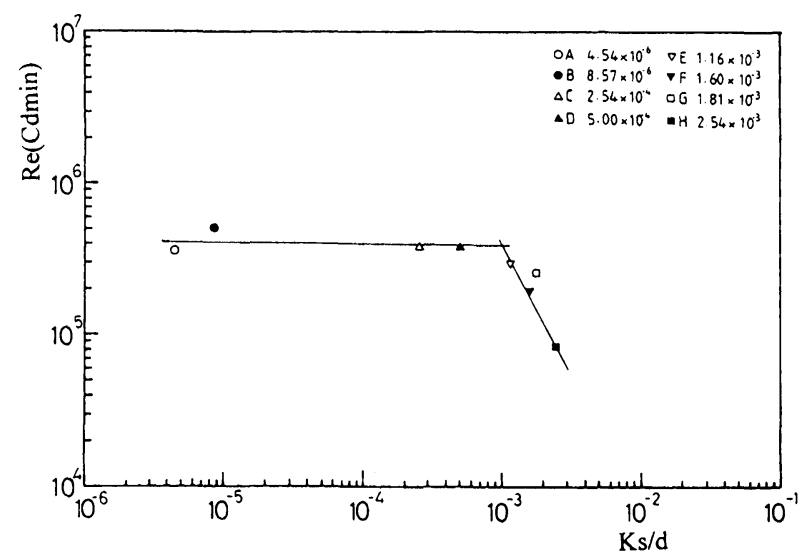

FIGURE 6 Variations of the Reynolds-number at critical point $\operatorname{Re}\left(C_{d \text { min }}\right)$ with relative roughness $\left(K_{s} / d\right)$.

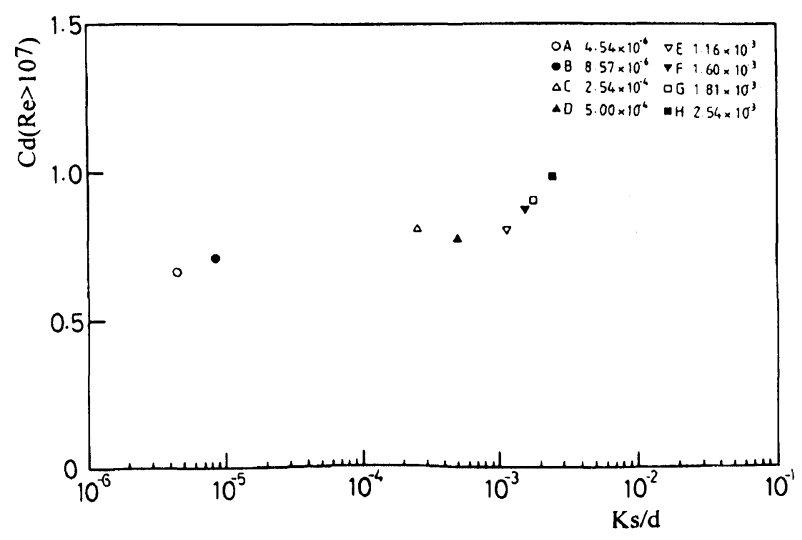

FIGURE 7 Variations of drag coefficient $C_{d}$ with relative roughness $\left(K_{s} / d\right)$ in the transcritical Reynolds-number range.

assumption is made that the vortex street drag may be equated to the cylinder drag. Thus it may be written:

$C_{D S} l=C_{D} d$

which, when multiplied by $f / U_{\infty}$, and rembering that $f=\left(U_{\infty}-u\right)$, may be reduced to the following:

$C_{D S}\left(1-\frac{u}{U_{\infty}}\right)=C_{D} S_{t}$

Using this calculated value of $C_{d} \cdot S_{t}\left[=C_{d s}\left(U_{\infty}-u\right) / U_{\infty}\right]$ and getting the corresponding value of $\left[\left(U_{\infty}-u\right) h / U_{\infty} l\right]$ in Figure 1 of the reference (Simmons 1975), we can calculate Bearman's number by the following equation:

$$
S_{B}=\frac{f h}{U_{s}}=\frac{f l}{k} \frac{h}{l U_{\infty}}=\frac{\hat{l}}{k} \frac{h}{l} \frac{\left(U_{\infty}-u\right)}{U_{\infty}}
$$

Figure 8 shows relation of Bearman's number with Reynolds-number. It shows the uniform value throughout the Reynolds-number range. It also shows that the calculated values of Bearman's number were independent of the surface roughness $\left(K_{s} / d\right)$.

\section{The Drag Reduction of the Circular Cylinder with Grooves [Adachi, et al. (1993)]}

See the variation and values of cylinder B in Fig. 4 and Table 2. The drag coefficient $C_{d}$ of this cylinder was smaller than any other cylinders in the range $3 \times 10^{5}<$ $\operatorname{Re}<10^{8}$. The relative roughness of the cylinder $B$ was $8.57 \times 10^{-6}$ in the circumferential direction but about 2.5 times as large in the axial direction. Comparing its relative roughness with that of cylinder $\mathrm{A}$, it was about twice as large in the circumferential direction but about 4 times as large in the axial direction. Nevertheless, its drag coefficient took smaller values at the critical point 


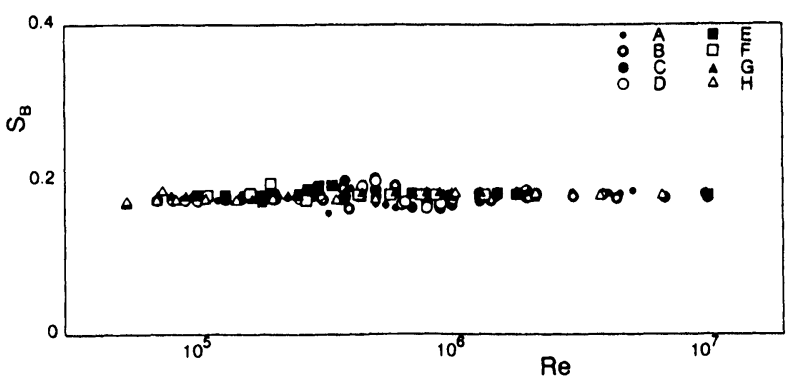

FIGURE 8 Variations of Bearman's number $S_{B}$ with Reynoldsnumber Re.

and supercritical Reynolds-number ranges, i.e., $4 \times 10^{5} \leqq \operatorname{Re} \leqq 10^{6}$. According to the researches about a flat plate placed parallel to the flow, the drag reduction as large as $8 \%$ was measured for a plate with longitudinal grooves having some particular dimensions, i.e., $\mathrm{h}^{+}=8$, $\mathrm{s}^{+}=16$ [Walsh, M.J., et al. $\left.(1980,1982)\right]$. This is called a riblet.

Here $\mathrm{h}^{+}$denotes the distance in the law of the wall variables

$h^{+}=h U_{\tau} / v$

$U_{\tau}=\sqrt{\tau_{W} / \rho}$

where

$U_{\tau}=$ friction velocity

$\tau_{w}=$ skin friction

$\rho=$ density

There are very few experimental results of skin friction along the cylinder surface available. Achenbach, E. $(1968,1971)$ measured the local transition skin friction of $\left(\tau_{w} / \rho U_{\infty}{ }^{2}\right)=0.005$ at the critical point and in the supercritical Reynolds-number range. Using thivalue, the height of groove $h^{+}$corresponding to the value of the distance in the law of wall variables is inversely proportional to the Reynolds-number. Its value is $14 \mu \mathrm{m}$ at $\operatorname{Re}=5 \times 10^{5}$ and $7 \mu \mathrm{m}$ at $\operatorname{Re}=10^{6}$. Then the relative roughness corresponding to the diameter $d=50 \mathrm{~mm}$ is $(h / d)=2.8 \times 10^{-5}$ and $1.4 \times 10^{-5}$, respectively. If $\mathrm{s}$ circumferential grooves having a width and depth of this value are engraved along the surface of a cylinder, they will serve as riblets and retard the transition of the flow. Then the drag reduction is expected.

To make a cylinder model with such surface grooves was difficult. The dimensions of the grooves engraved were as shown in Table 3, i.e., I, J, K, and L. We designate the depth of the groove as $h$, and the width as $s$. They were measured by tracing a stylus along the surface using a roughness meter. The measurement of roughness in circumferential direction $K_{s t}$ is based on JIS(Japanese Industrial Standard) B 0601: Definition and Designation of the Surface Roughness.

Figure 9 shows the variations of the drag coefficient with the Reynolds-number. In the subcritical Reynoldsnumber range, i.e. $\mathrm{Re} \leqq 3.8 \times 10^{5}$, there couldn't be seen no difference in the pressure distributions and drag coefficients. The effects of grooves were remarkable at the critical Reynolds-number and in the supercritical Reynolds-number range, i.e., $4 \times 10^{5} \leqq \mathrm{Re} \leqq 10^{6}$, as shown in Fig. 10a, b. A marked drag reduction could be seen in these ranges. Figure 11 shows the variations of the ratio of the drag coefficient $\left(C_{d} / C_{d s m o o t h}\right)$ with the Reynolds-number, where $C_{d s m o o t h}$ denotes the drag coefficient of the Cylinder A, i.e., the smoothest one. Reduction in the drag coefficient could be seen in the Reynoldsnumber range of $4 \leq 10^{5} \leqq \mathrm{Re}$. The maximum drag reduction ratio of each cylinder is $24 \%$ for B, $17 \%$ for I, $28 \%$ for $\mathrm{J}, 35 \%$ for $\mathrm{K}$ and $42 \%$ for $\mathrm{L}$. As the depth of grooves became shallower, the minimum values of the drag coefficient became smaller in the same order of the depth from $\mathrm{I}$ to $\mathrm{L}$. It took the minimum value at $\mathrm{L}$, and then took a larger value at $B$. Figure 12 shows the variations of base pressure coefficient $C_{p b}$ with the Reynolds-number for these cylinders, where base pressure was the average pressure value of 5 points which were $5^{\circ}$ apart taking that of $\theta=180^{\circ}$ as the center. As shown in this figure, a remarkable rise in the base pressure coefficient could be seen for Cylinders I, J, L and $\mathrm{B}$. But for the Cylinder $\mathrm{K}$, which showed a drag reduction in the widest Reynolds-number range, the values of $C_{p b}$ took somewhat lower values. Figure 13

TABLE 3

Dimensions of the grooved cylinders

\begin{tabular}{|c|c|c|c|c|c|c|}
\hline & $\mathrm{A}$ & $\mathrm{B}$ & $\bar{I}$ & $\bar{J}$ & $\mathrm{~K}$ & $\mathrm{~L}$ \\
\hline diameter $d(m)$ & $50 \leq 10^{-3}$ & $50 \leq 10^{-3}$ & $50 \leq 10^{-3}$ & $50.7 \leq 10^{-3}$ & $50 \leq 10^{-3}$ & $50.4 \leq 10^{-3}$ \\
\hline depth of groove $h(m)$ & $(0.03 \sim 0.5) \leq 10^{-6}$ & $(0.9 \sim 1.4) \leq 10^{-6}$ & $8.39 \leq 10^{-6}$ & $6.89 \leq 10^{-6}$ & $4.49 \leq 10^{-6}$ & $1.06 \leq 10^{-6}$ \\
\hline pitch of groove $s(m)$ & - & $47.5 \leq 10^{-6}$ & $35.5 \leq 10^{-6}$ & $35.4 \leq 10^{-6}$ & $38 \leq 10^{-6}$ & $24.4 \leq 10^{-6}$ \\
\hline relative depth of groove $(h / d)$ & $5.4 \leq 10^{-6}$ & $20 \leq 10^{-6}$ & $167.8 \leq 10^{-6}$ & $135.9 \leq 10^{-6}$ & $89.8 \leq 10^{-6}$ & $21 \leq 10^{-6}$ \\
\hline relative pitch of groove $(s / d)$ & - & $950 \leq 10^{-6}$ & $710 \leq 10^{-6}$ & $698.2 \leq 10^{-6}$ & $760 \leq 10^{-4}$ & $484.1 \leq 10^{-6}$ \\
\hline roughness $K s t$ & $(0.08 \sim 0.5) \leq 10^{-6}$ & $(0.3 \sim 0.5) \leq 10^{-6}$ & $1.98 \leq 10^{-6}$ & $4.3 \leq 10^{-6}$ & $2.5 \leq 10^{-6}$ & $0.2 \leq 10^{-6}$ \\
\hline relative surface roughness $(K s t / d)$ & $4.54 \leq 10^{-6}$ & $8.57 \leq 10^{-6}$ & $39.6 \leq 10^{-6}$ & $84.81 \leq 10^{-6}$ & $50 \leq 10^{-6}$ & $3.97 \leq 10^{-6}$ \\
\hline
\end{tabular}




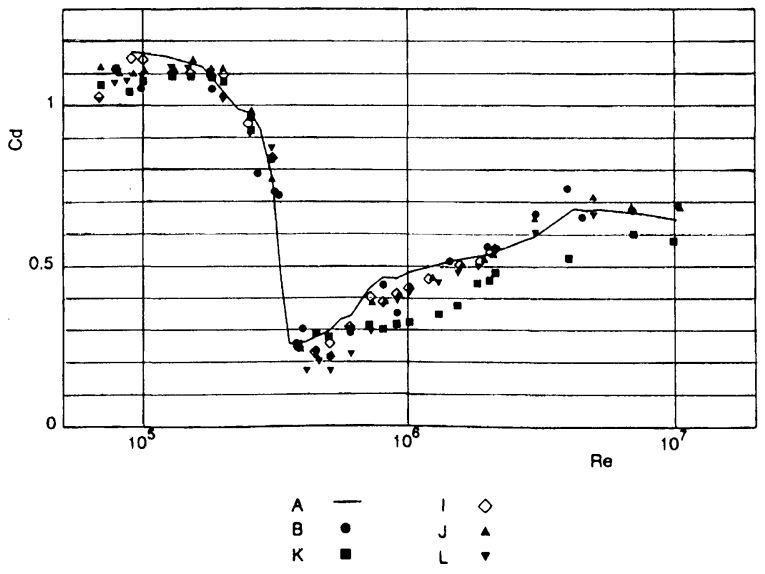

FIGURE 9 Variations of drag coefficient $C_{d}$ with Reynolds-number Re for the cylinder with grooves.

shows the variations of $\theta_{s p}$, i.e., the angle corresponds to the separation point, with the Reynolds-number. In Figures $13,14,15,16$, the values in the ordinate show the mean value of both sides of a cylinder. Figure 14 shows the variations of angle $\theta_{C p=0}$, which corresponds to pressure coefficient $C_{p}=0$, with the Reynolds-number. In the subcritical range, i.e., $\operatorname{Re} \geqq 2 \times 10^{5}$, no large differences can be seen, i.e., the pressure coefficient takes zero at $\theta=30^{\circ} \sim 34^{\circ}$. Cylinder $\mathrm{K}$ pressure coefficient became zero at $\theta=30^{\circ}$ in the wide range of supercritical and transcritical Reynolds-number ranges. The pressure distribution of Cylinder $\mathrm{K}$ shows a similar variation to that of the potential flow. No differences were seen in Cylinders I, J, L, and B, when compared to that of A. Fig. 15 shows the variations of the minimum value of pressure coefficient $C_{p \text { min }}$ and Fig. 16 shows those of the angle which corresponds to the minimum value of the pressure coefficient with the Reynolds-number.

From these it became clear that there were two causes of drag reduction. One was remarkable for Cylinders I, J, $\mathrm{L}$, and B, as shown in Fig. 12. The rises in base pressure often accompany a shift of separation point to downstream. These are seen in Cylinders I, L, B, and K. For Cylinder $\mathrm{K}$, the shift of the separation point to downstream could be seen in a comparatively wide Reynoldsnumber range, i.e., $5 \times 10^{5} \leqq \operatorname{Re} \leqq 10^{6}$. The other was the drop of pressure along the cylindrical surface. These characteristics were remarkable for Cylinder K. It could be considered that the laminar boundary layers were kept downstream along the surface in this case.

The fact that angle $\theta_{c p=0}$ which corresponded to the pressure coefficient $C_{p}=0$ took $30^{\circ}$ when $\operatorname{Re} \geqq 5 \times 10^{5}$ and the minimum value of pressure coefficient $C_{p \text { min }}$ -2.8 in a wide Reynolds-number range, i.e., this value

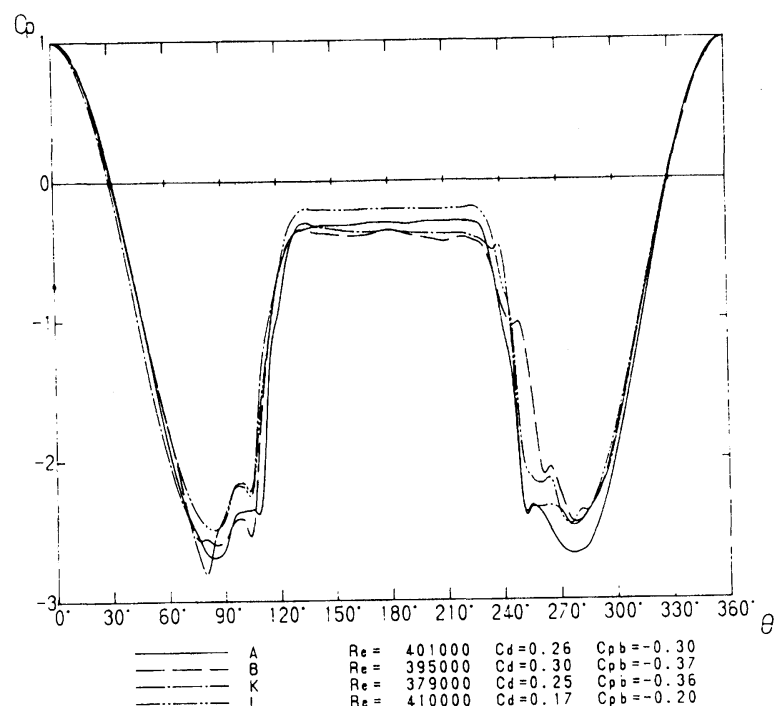

a) at the critical point

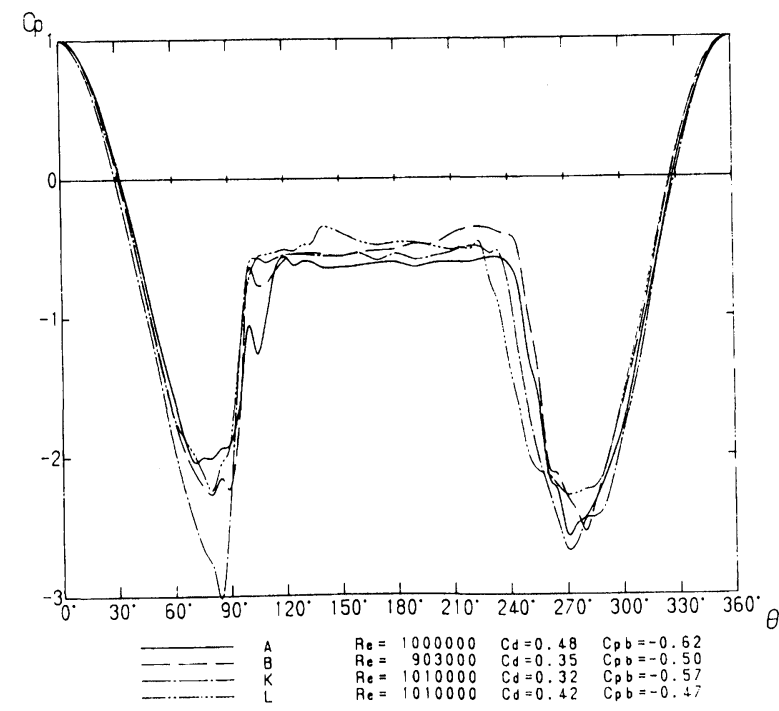

b) at the supercritical Reynolds-number range

FIGURE 10 Pressure distributions around the cylinder with grooves.

was very near to -3.0 of the potential flow, and the angle $\theta_{c p=\text { min. }}$, which corresponded to the minimum value of the pressure coefficient, was several degrees upstream from $\theta=90^{\circ}$ was remarkable for Cylinder K. Cylinders B, $\mathrm{K}, \mathrm{I}, \mathrm{J}$ and $\mathrm{L}$, the angle $\theta_{c p=\min }$. which corresponded to the minimum value of the pressure coefficient were somewhat downstream from that of A. If the negative values of the pressure coefficient were taken for $\theta \geqq 90^{\circ}$, the drag increased. There were differences in the pressure 


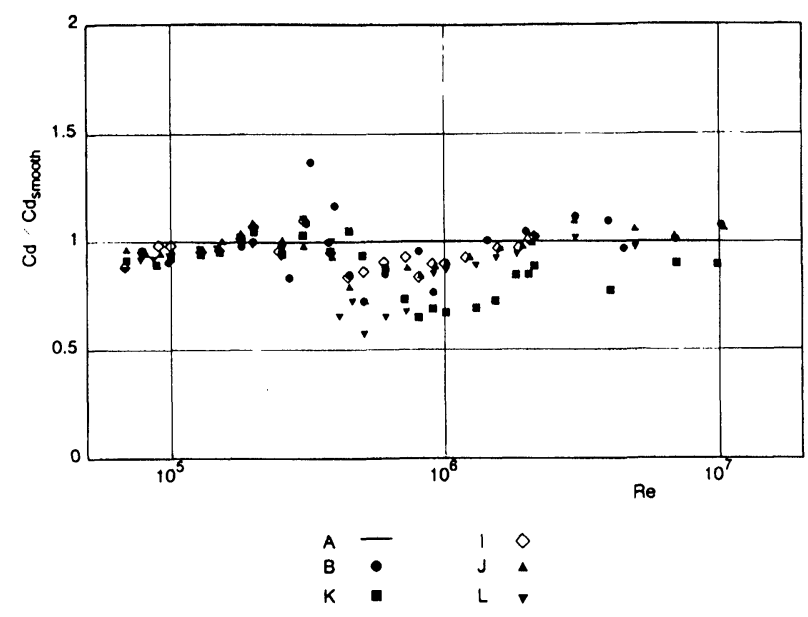

FIGURE 11 Variations of the ratio of the drag coefficient $\left(C_{d}\right)$ $\left.C_{d s m o o t h}\right)$ with the Reynolds-number.

distribution of Cylinder $\mathrm{K}$ from that of $\mathrm{A}$ in the transcritical Reynolds-number range. Especially for Cylinder K, the minimum value of the pressure coefficient became smaller and the angle corresponding to zero value of the pressure coefficient became $\theta=30^{\circ}$. Then the pressure distribution resembled that of the potential flow in shape.

By using the definition of pressure coefficient $C_{p}$, the drag coefficient $C_{d}$ can be written in the form

$$
\begin{aligned}
C_{d} & =\int_{0}^{\pi 2} C_{p} \cos \theta d \theta+\int_{\pi 2}^{\pi} \mathrm{C}_{p} \cos \theta d \theta \\
& =\int_{0}^{\pi / 2} \mathrm{C}_{p} d(\sin \theta)+\int_{\pi / 2}^{\pi} \mathrm{C}_{p} d(\sin \theta)
\end{aligned}
$$

Figures $17 \mathrm{a}, \mathrm{b}$ show the variations of the average values of the pressure coefficient of both sides in Fig. 10 with $\sin \theta$. The differences in the first and the second terms of Eq. (8) corresponded to the drag and thrust

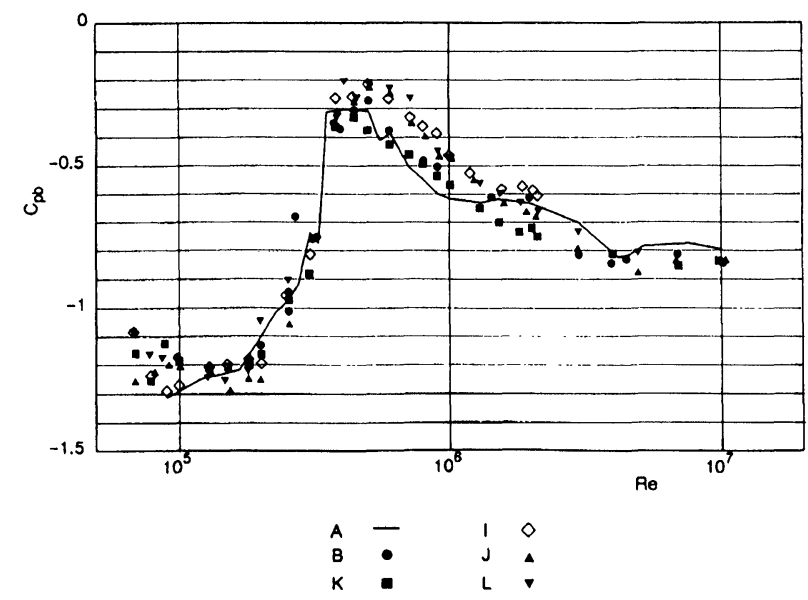

FIGURE 12 Variations of the base pressure coefficient, i.e., $C_{p b}$ with the Reynolds-number.

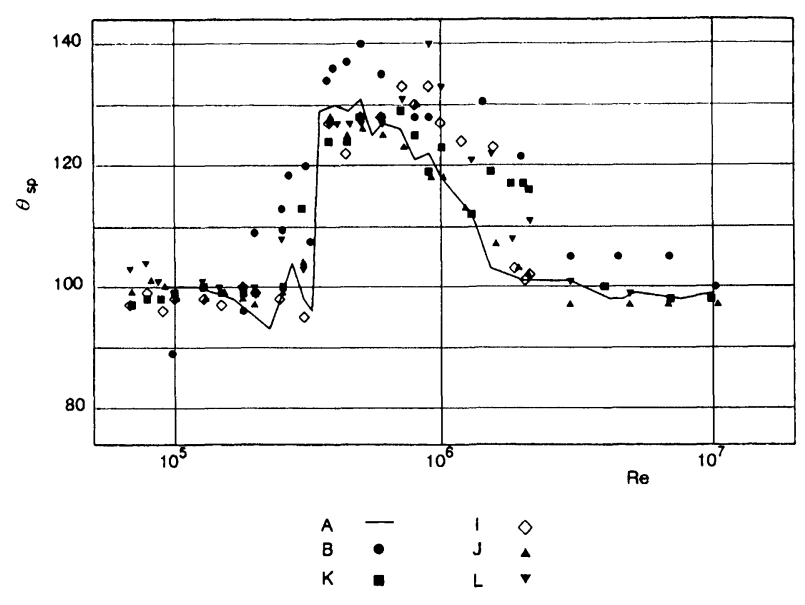

FIGURE 13 Variations of $\theta_{s p}$, i.e., the angle corresponding to the separation point, with the Reynolds-number.

(force directed forward), as shown in Fig. 17 by the hatched areas, respectively. This figure shows the contributions of the areas to the drag and to the thrust. In this figure, dotted lines show the potential flow for $\theta=0 \sim \pi / 2$. A decrease in drag corresponded to the increase in area of the thrust compared to the drag. Comparing Figures $\mathrm{K}$ and L, a decrease in drag was acquired, 1) because the pressure distribution approached that of potential flow for Cylinder K. 2) because the base pressure rises for Cylinder L. Then the reduction in drag was more remarkable at the critical point of Cylinder $\mathrm{L}$, and in the supercritical Reynolds-number range of cylinder $\mathrm{K}$.

The effect of the riblet was to modify and reduce the momentum exchange properties caused by the streamwise vortices developing near the surface beneath a turbulent boundary layer with a consequent reduction in the surface shear stress. This process enabled the riblet

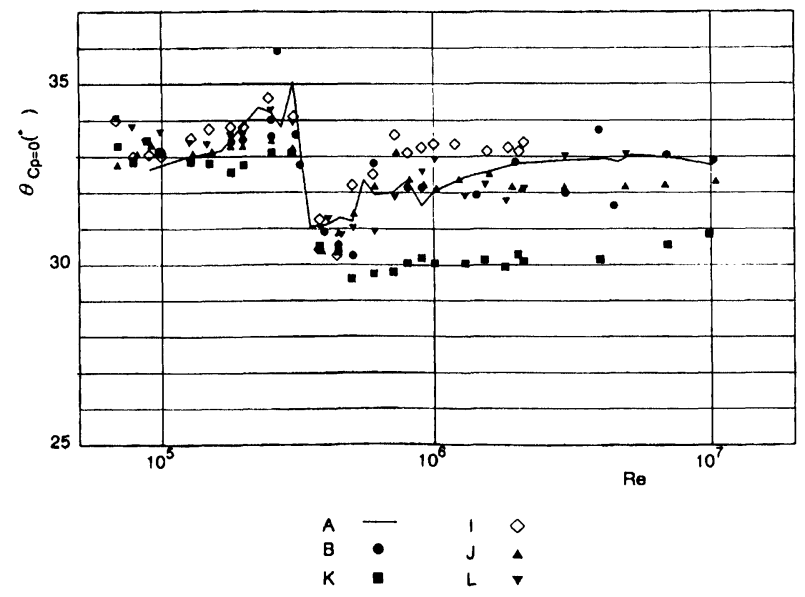

FIGURE 14 Variations of Angle $\theta_{C p=0}$ with the Reynolds-number. 


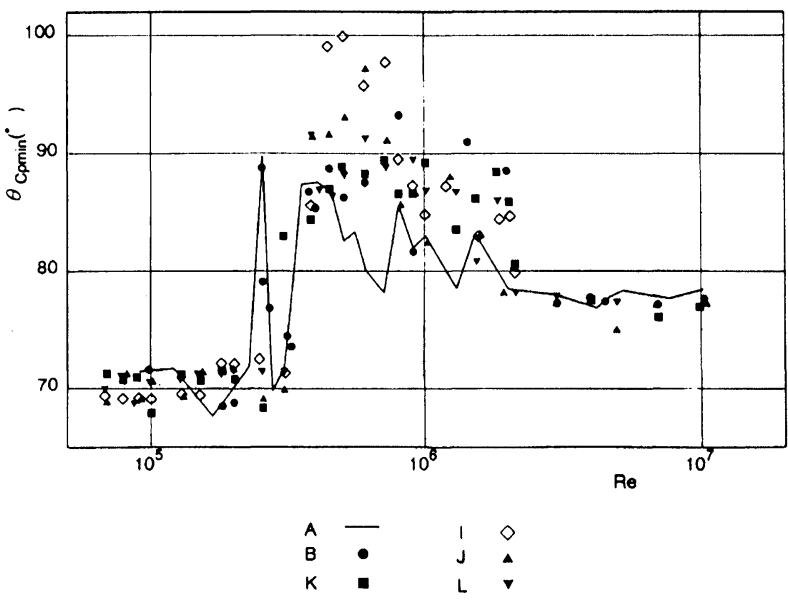

FIGURE 15 Variations of the minimum value of pressure coefficient, i.e., $C_{p}$ min with the Reynolds-number.

surface to retard the development of the turbulent boundary layer and then to retard the development of the turbulent boundary layer and thus to reduce the surface drag. The shifting of the separation point downstream and the rising of base pressure of Cylinders I, J, L and B was considered to be this effect. For Cylinder $\mathrm{K}^{\prime}$ to keep laminar boundary layers along both sides and consequently to make pressure lower were the causes of the reduction in the drag. In this case, the reduction in drag has been acquired in a more wide Reynolds-number range.

\section{Reduction of the Drag on the HARLOP}

Making an airship staying at a point in the stratosphere, i.e., at the height of $20 \mathrm{~km}$, was planned. It served as an observation balloon to the environment, and a junction point for the communication, etc.. The airship was called

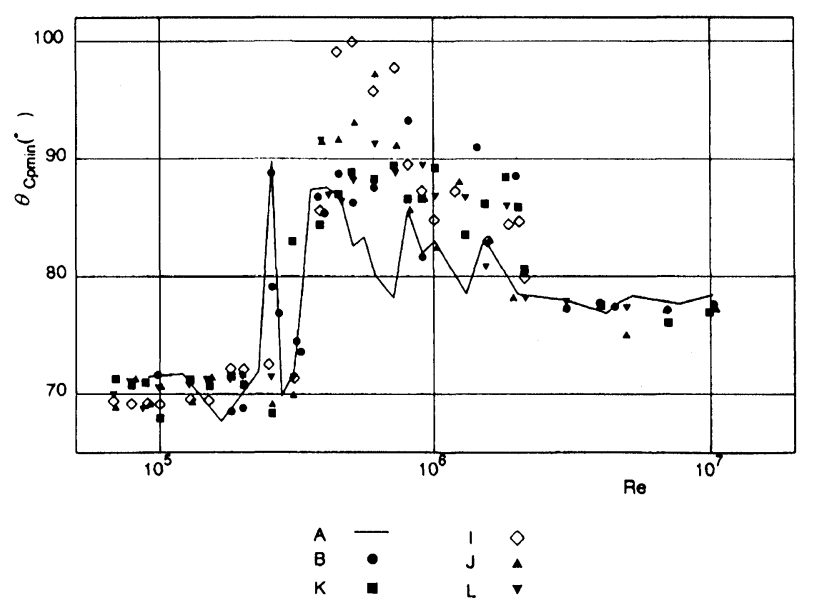

FIGURE 16 Variations of the angle corresponding to the minimum pressure coefficient, i.e., $\theta_{p \text { min }}$, with the Reynolds-number.
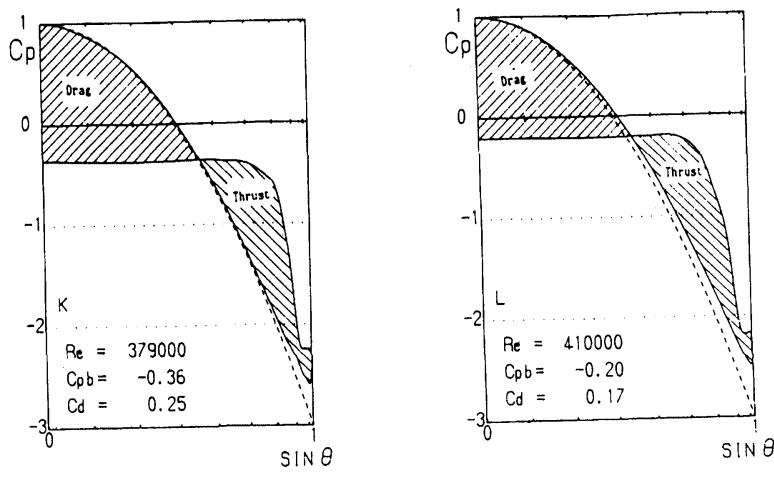

a) In the neighborhood of the critical point
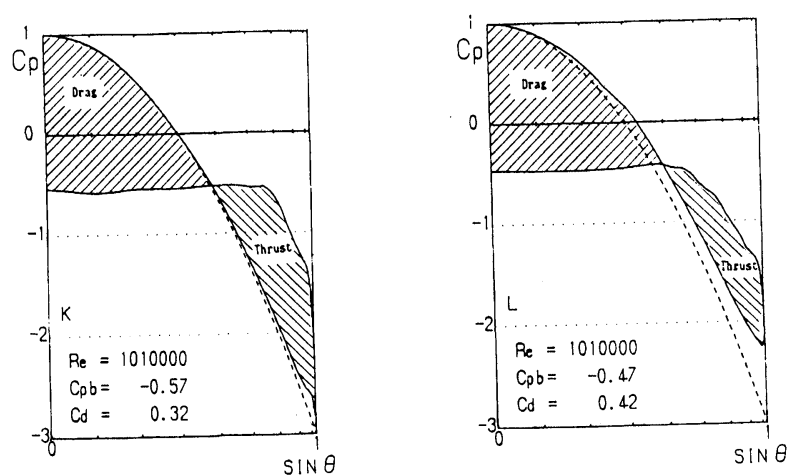

b) In the supercritical range

FIGURE 17 Drag and thrust.

HARLOP (High Altitude Long Range Observation Platform). At this high altitude, the prevailing westerlines are the most weak, but it sometimes becomes became 40 $\mathrm{m} / \mathrm{s}$. In order to make the balloon stay at a point, it must have a motor driven by a solar battery, but the drag acting on the body must be small. Figure 18 shows its general view and Table 4 shows its specifications. The flow around the body is a high Reynolds and low Machnumber flow.

In order to study the mechanism of the drag acting on the body, some measurements were made using a wind tunnel model as shown in Fig. 19. Dividing the body into two parts, i.e., from the top to the maximum diameter and hereafter, the form drag can be calculated for the former part

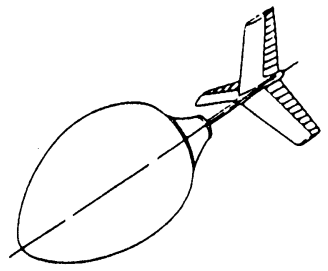

FIGURE 18 The general view of HARLOP. 
TABLE 4

The Specifications of HARLOP

\begin{tabular}{ll}
\hline Attitude & $20,000 \mathrm{~m}$ \\
Length of body & $\mathrm{L}=185 \mathrm{~m}$ \\
Flow velocity & $40 \mathrm{~m}$ \\
Mach-number & 0.14 \\
Reynolds-number & $1.85 \times 10^{7}$ \\
\hline
\end{tabular}

$$
\begin{aligned}
D_{1}= & \pi \int_{0}^{l}(1 / 2) \rho U_{\infty}{ }^{2} C_{p} R^{2} d(r / R)^{2}+ \\
& \pi \int_{0}^{l}(1 / 2) \rho(R H O) U_{\infty}{ }^{2} C_{p} R^{2} d(r / R)^{2}
\end{aligned}
$$

and for the latter part

$$
\begin{aligned}
D_{2}= & \pi \int_{l}^{h / R}(1 / 2) \rho U_{\infty}{ }^{2} C_{p} R^{2} d(r / R)^{2}+ \\
& \pi \int_{l}^{h / R}(1 / 2) \rho(R H O) U_{\infty}{ }^{2} C_{p} R^{2} d(r / R)^{2}
\end{aligned}
$$

Figure 20 shows relation between $C_{p}$ and $(r / R)^{2}$ at Reynolds-number $2.09 \times 10^{6}$ and $3.34 \times 10^{6}$ for the former and latter parts, respectively. The total form drag consists of the two parts, i.e., $D_{p}=D_{1}+D_{2}$. Then the difference between the areas corresponds to the form drag. In this figure, the broken line shows the calculated value by Goldschmied (1987). With suction, the pressure distribution was denoted by the dotted line.

\section{CONCLUSIONS}

In this paper, first the special features of our cryogenic wind tunnel were described. By making pressure in the wind tunnel high and then making temperature low, we attained a high Reynolds-number, but a low Machnumber flow. Applying inner insulating principles, we

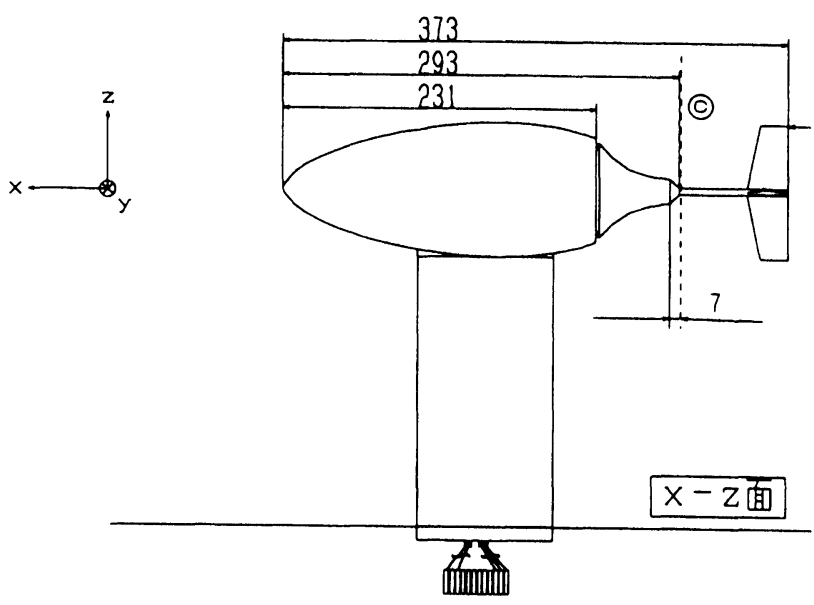

FIGURE 19 The wind tunnel model of HARLOP.

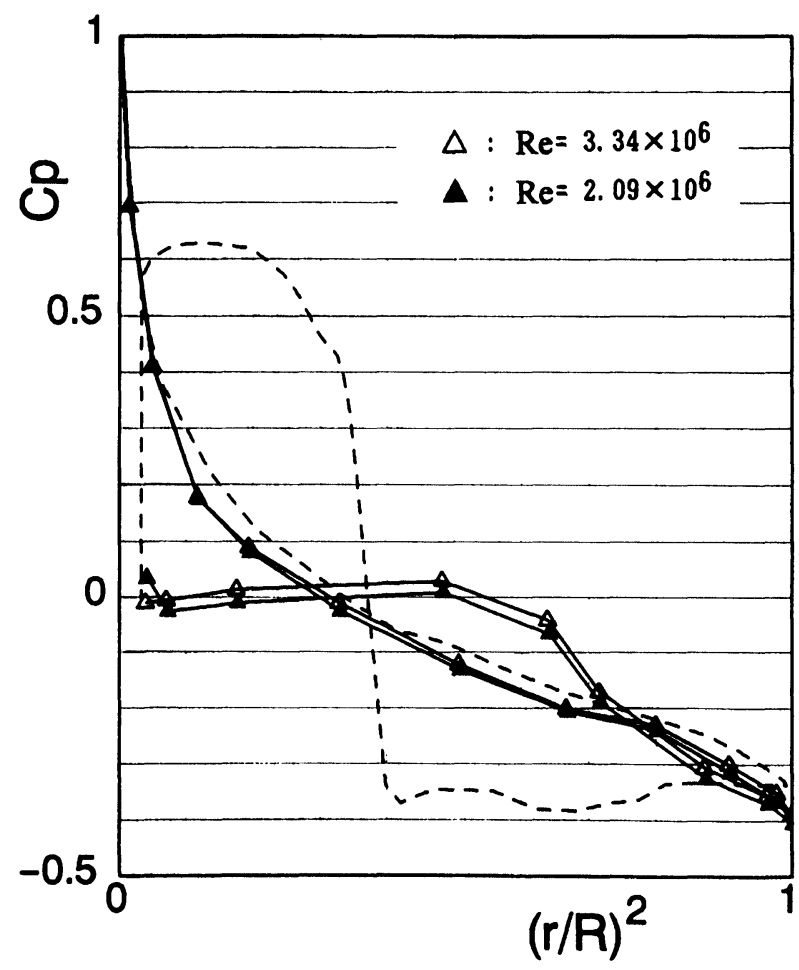

FIGURE 20 The drag and thrust of HARLOP.

attained a high Reynolds-number flow with a comparatively small consumption of liquid nitrogen.

The effects of surface roughness on the drag were investigated using a circular cylinder with various surface roughnesses. The value of relative roughness affected the Reynolds-number range in which flow ranges existed. There was a limiting value of surface roughness $\left(K_{s} / d\right)$, which influenced the value of the Reynoldsnumber at the critical point. If the relative surface roughness was smaller than this limiting value, the variation of $C_{d}$ with the Reynolds-number showed the same trend in the smaller Reynolds-number at the critical point. For a cylinder with larger surface roughness than this critical value, the Reynolds-number range than the critical point became smaller with an increase in $\left(K_{s} / d\right)$. In these super and transcritical Reynolds-number ranges, there was not such a limiting value.

The universality of the Strouhal-number in the wake of cylinders with various surface roughness was considered. Using the measured values of frequency of the vortex street, pressure distribution and drag coefficient, the universal Strouhal-numbers were calculated. It was made clear that Bearman's Number had the most uniform value throughout the Reynolds-number range. It was also shown that Bearman's number was independent from the surface roughness. 
A study of the effects of V-grooves on the drag reduction of a circular cylinder was conducted. With reference to the results about riblets, which were made on the plate placed parallel to the stream, the size of the $\mathrm{V}$-groove was determined. Circular cylinders with various V-groove were tested and acquired the maximum drag decrease of $42 \%$. The effects of V-grooves were classified into two cases. One case showed the original effects of the riblet, i.e., to suppress bursting and turbulent separation. In the other case, V-gro.oves contributed to keeping a laminar boundary layer along the surface and pressure distribution around the surface closely resembling that of the potential flow.

A model test of HARLOP was planned. From the results without suction, it is prospected to decrease the drag with suction of the boundary layer.

\begin{tabular}{|c|c|c|}
\hline \multicolumn{3}{|c|}{ Nomenclature } \\
\hline$C_{d}$ & $:$ & drag coefficient $\left[=(1 / 2) \int^{2 \pi} \mathrm{C}_{p} \cos \theta d \theta\right]$ \\
\hline$C_{p}$ & $:$ & pressure coefficient $\left[=\left(p_{0}^{0}-p_{x}\right) /\left(\rho U_{x}^{2} / 2\right)\right]$ \\
\hline$C_{p b}$ & $:$ & back pressure coefficient \\
\hline$d$ & $:$ & $\begin{array}{l}\text { reference length or length of the model (for } \\
\text { cylinder: diameter) }\end{array}$ \\
\hline$h$ & $:$ & depth of the grooves \\
\hline$(h / d)$ & : & relative depth of the grooves \\
\hline$K_{s}$ & : & roughness \\
\hline$\left(K_{s} / d\right)$ & $:$ & relative roughness \\
\hline$n$ & $:$ & vortex shedding frequency \\
\hline$p$ & : & pressure \\
\hline$R_{e}$ & $:$ & Reynolds-number $\left(=U_{x} d / v\right)$ \\
\hline$s$ & $:$ & pitch of the grooves \\
\hline$T$ & $:$ & temperature \\
\hline$U$ & $:$ & velocity \\
\hline$\rho$ & $:$ & density \\
\hline$\mu$ & $:$ & viscosity \\
\hline$v$ & $:$ & kinetic viscosity \\
\hline
\end{tabular}

\section{Subscripts}

$a$
$b$
crit
min
$t$
$\infty$

axial-direction

suction or back side

critical value

minimum value

circumferential direction

undisturbed flow

\section{References}

Achenbach, E., 1968, "Distribution of local pressure and skin friction around a cylinder in cross-flow up to $\operatorname{Re}=5 \leq 10^{6}$ ", Journal of Fluid Mechanics, Vol. 34, Part 4, pp 625-639.

Achenbach, E., 1971, "Influence of surface roughness on the cross-flow around a cylinder", Journal of Fluid Mechanics, Vol. 46, Part 2, pp 321-335.

Adachi, T, Ono, H., Matsuuchi, K., Kawai, T., and Cho, T., 1989a, "Flow around a circular cylinder in the High Reynolds-number Range (Effect of Surface Roughness)", Trans. Jpn. Soc. Mech. Eng., (in Japanese), Vol. 55, No. 511, 685-692.

Adachi, T., Ono, H., Matsuuchi, K., Kawai, T., and Cho, T., 1989b, "Drag and Vortex Shedding from Circular Cylinder in the High Reynolds-number Range (Effect of Surface Roughness)", Trans. Jpn. Soc. Mech., (in Japanese), Vol. 55, No. 517, 2597-2601.

Adachi, T., Matsuuchi, K., and Kawai, T., 1990a, "Effect of Surface Roughness on the Drag and Vortex Shedding from a Circular Cylinder", Proc. of The Second KSME/JSME Fluids Engineering Conference, Vol. 1, Oct, 10-13, 78-83.

Adachi, T., Ono, T., Matsuuchi, K., Kawai, T., and Maeda, H., 1990b, "The Effects of a Wake Splitter Plate on the Flow around a Circular Cylinder", Trans. Jpn. Soc. Mech., (in Japanese), Vol. 56, No. 528, 2225-2232.

Adachi, T., Matsuuchi, K., and Kawai, T., 1991, "High Reynoldsnumber flow experiments using cryogenic wind tunnel", Proc. of The ASME/JSME Thermal Engineering Joint Conference", Vol. 5, 371-378.

Adachi, T., Maeda, H., Shiono, M., Ozaki, T., Matsuuchi, K., and Kawai, T., 1993, "Drag reduction of circular cylinder in the highReynolds-number range”, Trans. Jpn. Soc. Mech. Eng., (in Japanese), Vol. 59, No. 558.

Bearman, P. W., On vortex street wakes, J. Fluid Mech., 28-4 (1967), 625 .

Goldschmied, F. R., 1987, "Fuselage Self-Propulsion by Static Pressure Thrust: Wind Tunnel Verification", AIAA/AHS/ASEE Aircraft Design, Systems and Operations Meeting, Sept. 14-16, 1-20.

Griffin, O. M., Universal Similarity in the Wakes of Stationary and Vibrating Bluff Structures, Trans. ASME, J. Fluid Engng., 103-1 (1981), 52.

Roshko, A., Experiments on the flow past a circular cylinder at very high Reynolds-number, J. Fluid Mech., 10-3 (1961), 345.

Simmons, J. E. I., Effect of Separation Angle on Vortex Streets, Proc. Am. Soc. Eng., J. Eng. Mech. Div., 1975-10, 649.

Walsh, M. J., 1980, "Drag Characteristics of V-groove and Transverse Curvature Riblets", AIAA Progress in Astronautics and Aeronautics, Vol. 72, 168-184.

Walsh, M. J., 1982, "Turbulent Boundary Layer Drag Reduction Using Riblets", AIAA 20th Aerospace Sciences Meeting, AIAA-82-0169, $1-6$. 

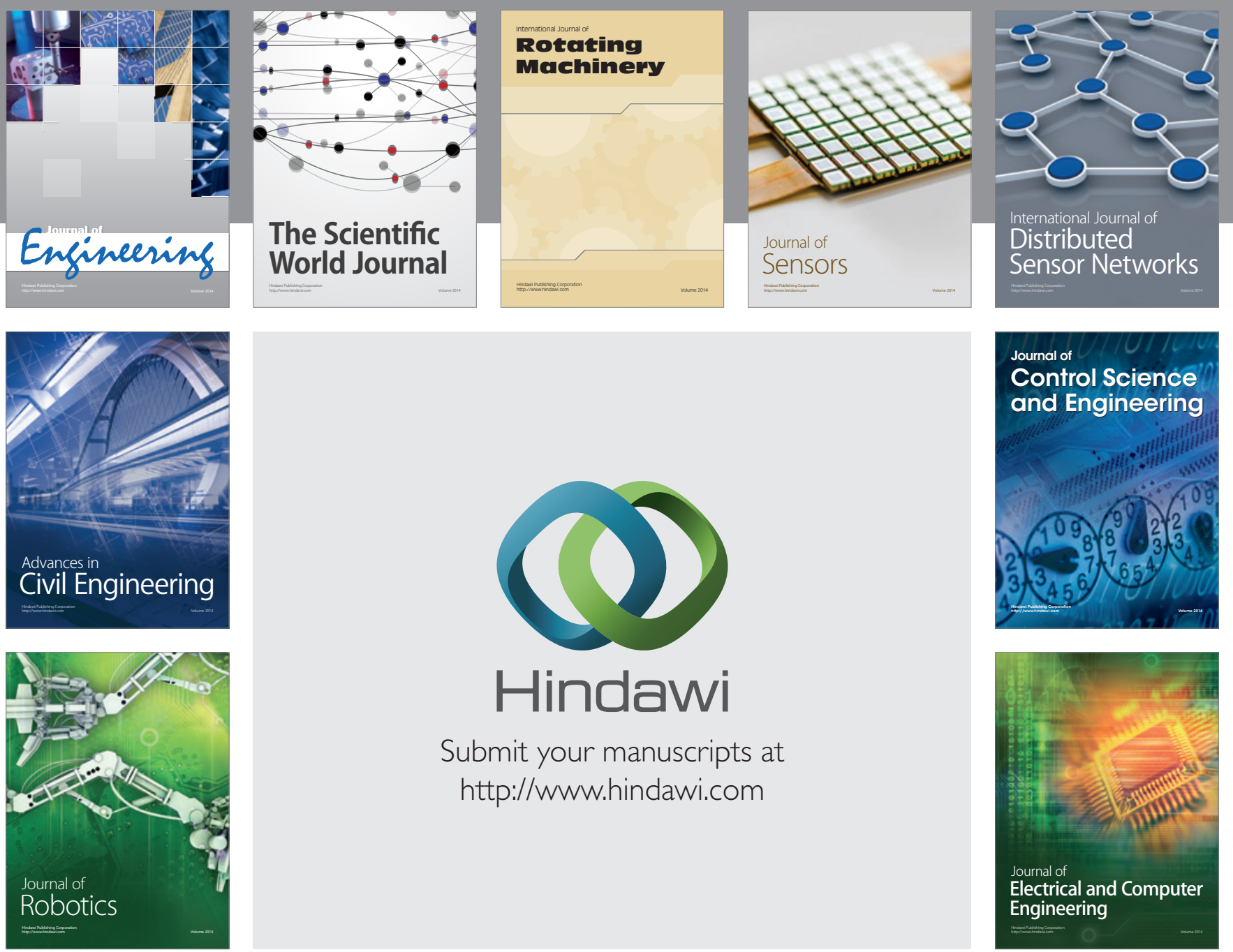

Submit your manuscripts at

http://www.hindawi.com
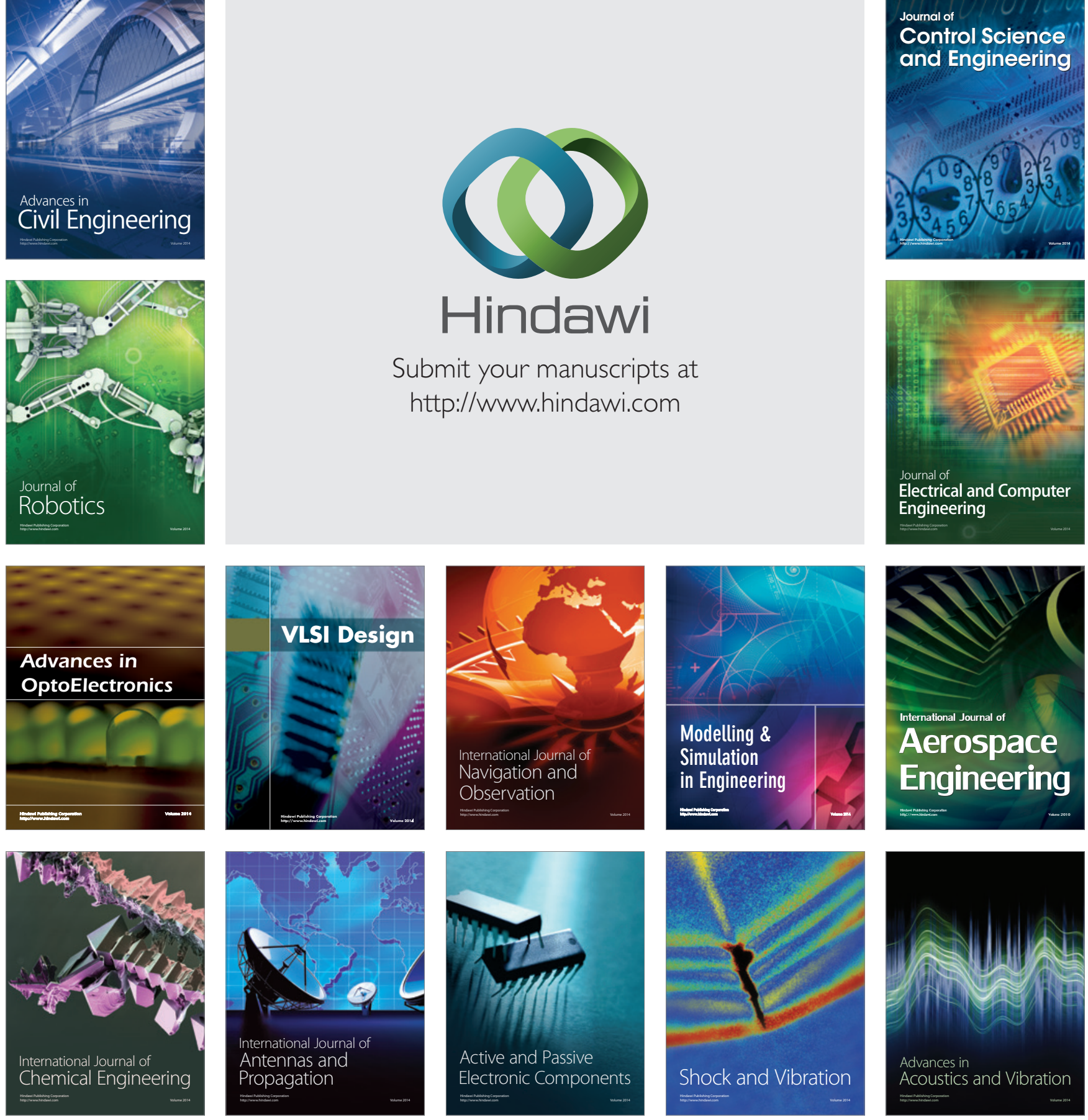